\title{
Motor sequences; separating the sequence from the motor. A longitudinal rsfMRI study
}

\author{
Anna-Thekla P. Jäger ${ }^{1,2}$ (1) Julia M. Huntenburg ${ }^{3} \cdot$ Stefanie A. Tremblay ${ }^{4,5} \cdot$ Uta Schneider $^{1} \cdot$ Sophia Grahl $^{6}$. \\ Julia Huck $^{4}$. Christine L. Tardif ${ }^{7,8}$. Arno Villringer ${ }^{1,2,9,10,11}$. Claudine J. Gauthier ${ }^{4,5}$. Pierre-Louis Bazin ${ }^{1,12}$. \\ Christopher J. Steele ${ }^{1,13}$
}

Received: 16 February 2021 / Accepted: 8 October 2021 / Published online: 27 October 2021

(c) The Author(s) 2021

\begin{abstract}
In motor learning, sequence specificity, i.e. the learning of specific sequential associations, has predominantly been studied using task-based fMRI paradigms. However, offline changes in resting state functional connectivity after sequence-specific motor learning are less well understood. Previous research has established that plastic changes following motor learning can be divided into stages including fast learning, slow learning and retention. A description of how resting state functional connectivity after sequence-specific motor sequence learning (MSL) develops across these stages is missing. This study aimed to identify plastic alterations in whole-brain functional connectivity after learning a complex motor sequence by contrasting an active group who learned a complex sequence with a control group who performed a control task matched for motor execution. Resting state fMRI and behavioural performance were collected in both groups over the course of 5 consecutive training days and at follow-up after 12 days to encompass fast learning, slow learning, overall learning and retention. Between-group interaction analyses showed sequence-specific decreases in functional connectivity during overall learning in the right supplementary motor area (SMA). We found that connectivity changes in a key region of the motor network, the superior parietal cortex (SPC) were not a result of sequence-specific learning but were instead linked to motor execution. Our study confirms the sequence-specific role of SMA that has previously been identified in online task-based learning studies, and extends it to resting state network changes after sequence-specific MSL.
\end{abstract}

Keywords Motor sequence learning $\cdot \operatorname{rsfMRI} \cdot$ Sequence specificity

P. L. Bazin and C. J. Steele contributed equally to this work.

Anna-Thekla P. Jäger

annatheklajaeger@gmail.com

1 Department of Neurology, Max Planck Institute for Human Cognitive and Brain Sciences, Leipzig, Germany

2 Center for Stroke Research Berlin (CSB), CharitéUniversitätsmedizin Berlin, Berlin, Germany

3 Neuroscience Programme, Champalimaud Research, Lisbon, Portugal

4 Department of Physics/Perform Center, Concordia University, Montreal, QC, Canada

5 Montreal Heart Institute, Montreal, QC, Canada

6 Clinic of Neurology, Technical University Munich, Munich, Germany
7 Department of Biomedical Engineering, McGill University, Montreal, QC, Canada

8 Montreal Neurological Institute, Montreal, QC, Canada

9 Clinic for Cognitive Neurology, Leipzig, Germany

10 IFB Adiposity Diseases, Leipzig University Medical Centre, Leipzig, Germany

11 Collaborative Research Centre 1052-A5, University of Leipzig, Leipzig, Germany

12 Faculty of Social and Behavioral Sciences, University of Amsterdam, Amsterdam, Netherlands

13 Department of Psychology, Concordia University, Montreal, QC, Canada 


\section{Introduction}

Motor learning-induced brain plasticity has been typically studied using magnetic resonance imaging (MRI) (for review, see Dayan and Cohen 2011; Krakauer et al. 2019; Taubert et al. 2012), from immediate online functional changes (Coynel et al. 2010; Karim et al. 2017; Steele and Penhune 2010; Yokoi and Diedrichsen 2019) to long-term structural effects (Gryga et al. 2012; Taubert et al. 2012; Bengtsson et al. 2005; Scholz et al. 2009). While task-based studies investigate immediate trainingrelated processes during task performance and structural studies usually identify longer term changes in brain morphometry, they cannot comment on how the learned skill is represented and maintained within functional networks of the brain outside of the training/learning context. Resting state fMRI (rsfMRI) can be used to investigate functional plasticity that occurs between online functional and slower structural changes. Measured in the absence of a task, resting-state network dynamics are thought to reflect the previous co-activation of functionally connected brain regions (Biswal et al. 1995; Guerra-Carrillo et al. 2014). Alterations in these functional networks are thought to reflect the strengthening of the memory trace generated during practice (Albert et al. 2009; Lewis et al. 2009; Vahdat et al. 2011). Therefore, assessing resting state networks and how they change in response to training can provide unique insight into training-related functional plasticity beyond the immediate time point of learning.

Previous research in motor learning has established that sequence learning per se can be distinguished from motor execution (Rosenbaum et al. 1983; Penhune and Steele 2012; Wiestler and Diedrichsen 2013). However, while this concept has been assessed, tested and discussed in task-based studies (Seidler et al. 2002; Yokoi and Diedrichsen 2019; Wymbs and Grafton 2015), learning that is specific to sequential associations (i.e., sequence-specific learning) is rarely if ever differentiated from motor execution in rsfMRI studies of motor learning. In part, this is due to the added challenge of including additional control groups matched for motor execution. Conclusions about functional changes being the result of sequence-specific learning, and not simply an effect of repeated performance, cannot be made without the inclusion of a control group (Thomas and Baker 2013; Steel et al. 2019). As a result, it is currently unclear which regions of the sensorimotor network are involved in offline sequence-specific learning versus those more generally involved in motor execution.

RsfMRI connectivity changes after motor sequence learning (MSL) have been investigated with "pre/post" frameworks where resting state functional connectivity (rsFC) is typically measured only before and/or after performing the task to identify immediate and overnight effects (Sami and Miall 2013; Gregory et al. 2014; Sami et al. 2014; Mary et al. 2017; Albert et al. 2009). Also, while motor learning is thought to progress through several stages involving rapid changes in performance (fast learning), slower improvements over a longer time period (slow learning), and maintenance of robust performance (retention) (Doyon and Benali 2005; Dayan and Cohen 2011; Lohse et al. 2014), there are few studies that have followed changes in rsfMRI networks after MSL across multiple sessions of training to investigate larger timescales (Ma et al. 2011; Xiong et al. 2009). To our knowledge, there has been no investigation of sequence-specific resting state network plasticity across all three stages of learning. The present study is the first to address this gap.

Here, participants learned a continuous motor task with their right (dominant) hand over 5 days of training, followed by a retention probe 12 days later. RsfMRI was collected five times over the course of the training, providing a rich sampling of the different stages of learning in the absence of performance. Crucially, participants were randomly assigned to either a training (sequence-specific training) or control group (matched for motor execution). This rich dataset allowed us to identify the brain regions involved in sequence-specific learning and explore their functional dynamics.

\section{Methods}

\section{Participants}

Forty right-handed healthy participants with no history of neurological disorder (22 females, ranging from 20 to 32 years of age, $\mathrm{M} \pm \mathrm{SD}$ : $24.5 \pm 2.44$ ) were included in this study. Participants were recruited from the database of the Max Planck Institute for Human Cognitive and Brain Sciences in Leipzig, Germany, and randomly assigned to an experimental $(N=20,11$ females $)$ and control group $(N=20,10$ females $)$. The experimental group learned a previously published complex visuomotor sequence pinchforce task (Gryga et al. 2012) while the control group performed a much simpler sinusoidal sequence that required almost no learning to perform. The training period consisted of 5 days, with an additional familiarization session prior to training and a retention session following 12 days without practice. Participants did not meet any MRI exclusion criteria, gave written informed consent in accordance with the Declaration of Helsinki, and were monetarily compensated for their time. The study design was approved by the ethics review board of the Medical Faculty of the University of Leipzig. 


\section{Task and stimuli: sequential pinch force task}

The sequential pinch force task (SPFT) is a motor sequence task that requires the participant to use the index finger and thumb of the dominant (right) hand to exert force on the end of a pinch force device (Fig. 1a) which measures force at a sample rate of $80 \mathrm{~Hz}$. This pinching force controls the height of a rectangular yellow force bar (FOR) that is displayed on the screen (Fig. 1b).

In the SPFT, the participant is instructed to adjust the height of FOR to match that of an adjacent blue reference (REF) bar. The up and down movement of REF follows a pre-set sequence (Fig. 1b, c). There are 3 conditions: a learning condition (LRN), a simple control condition (SMP) and a resting condition (RST). In LRN, the movement of the bar follows a previously published sequence of varying heights that is difficult to predict (Fig. 1c) and learned over time (Gryga 2012), while the SMP sequence is a simple sinusoid. The SMP sequence was designed to match the LRN sequence for its frequency of maximum power, duration, range, and the total magnitude of force. While in the SMP condition participants merely performed this predictable and unvarying sinusoidal sequence of motor movements; in the LRN condition, participants execute the same type of movements (i.e., varying pinch force over time) but performed a more complex sequence (Fig. 1c). As a result, SMP serves as a matched control for motor execution which, in comparison with LRN, can be used to identify sequence-specific differences that are over and above any changes associated with motor execution. During RST, both bars are displayed statically at $50 \%$ of their maximum height and participants were instructed to focus their gaze between the upper edges of the two bars.

On each training day, 3 blocks consisting of 3 SMP, LRN and RST trials each were performed by the experimental group; for a total of 9 trials per condition on each day. In order to ensure that the training was the same on all days, the order was fixed across participants such that they started with the SMP trials followed by RST and then LRN trials. The control group performed the same number of blocks and trials but never performed LRN: the LRN blocks were replaced by SMP blocks such that the total effort for each group remained the same. Each trial was $18 \mathrm{~s}$ in length. The sequential force trace was the same across participants to ensure that all participants faced the same sequence difficulty. After the SMP and LRN trials, participants received feedback reflecting their average temporal accuracy in matching the heights of REF and FOR. The average temporal accuracy consisted of the mean time lag per block (in $\mathrm{ms})$.

For simplicity, we will refer to the groups based on which sequence they were assigned throughout the rest of this paper: SMP (SMP only) vs. LRN (SMP and LRN).

\section{Training and experimental procedure}

The experimental procedure (Fig. 1d) consisted of one familiarization session (d0), five training sessions (d1-d5) and one retention session (d17, which took place 12 days after $\mathrm{d} 5$ ). The first training session (d1) always a
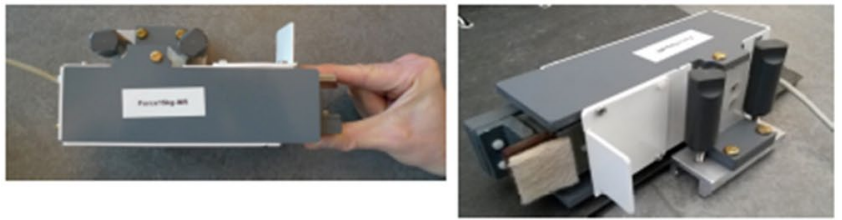

b

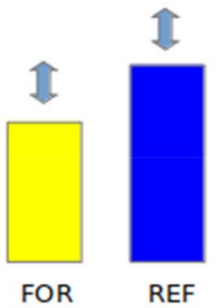

c

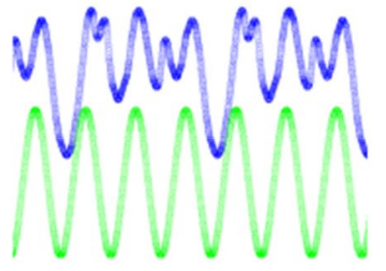

d

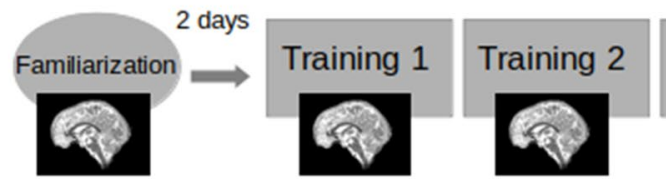

Training 3
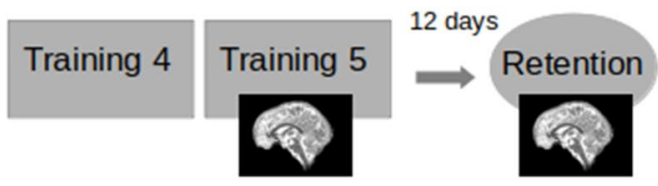

Fig. 1 Task and procedure-a sequential pinch force task (SPFT) device, b visual screen representations: FOR bar (yellow) REF bar (blue), c pinch-force task, green: simple control condition (SMP) sequence, blue: learning condition (LRN) sequence $\mathbf{d}$ experimental design. Brain images indicate sessions where $7 \mathrm{~T}$ scanning was performed 
took place on Monday and the d0 familiarization session took place on the previous Friday so that the training period for each participant always took place on five consecutive days (Monday-Friday). The familiarization session included a maximum force calibration step followed by 9 trials of the SMP sequence. When measuring the maximum pinch force, the subject was asked to pinch the device with as much force as possible for ten seconds and the maximum value obtained during this period was considered maximum force. This measurement was used to calibrate the level of force required to move the visually presented force bar and was set to between 5\% (minimum bar level) and 30\% (maximum bar level) of each individual's maximum force to ensure that participants were also matched by relative effort. Five sessions (d0, d1, d2, d5, d17) were performed while lying supine inside of the MRI scanner and two (d3, d4) outside of the scanner in a separate testing room while seated at a computer. During these sessions, the SPFT was performed with the dominant hand, over a total duration of 20 min (3 blocks, 9 trials [LRN group: $3 x(L R N), 3 x($ SMP), $3 x(R S T)$; SMP group: $3 x(\mathrm{SMP}), 3 x(\mathrm{SMP}), 3 x$ (RST)]. The $\mathrm{d} 17$ retention session followed the same protocol as the training days and was measured 12 days after the last day of the training period. All sessions took place at the same time during the day to account for potential time of day fluctuations in resting state connectivity (Steel et al. 2019).

\section{MRI protocol}

MRI data were acquired on a 7 Tesla MRI scanner (MAGNETOM, Siemens Healthcare, Erlangen, Germany) equipped with a 32-channel head coil (Nova). For the purposes of the current study, blood-oxygenation-level dependent (BOLD) rsfMRI, MP2RAGE T1 (Marques et al. 2010), and a fieldmap were acquired. RsfMRI scans $[$ BOLD, voxel dimensions $=1.2 \times 1.2 \times 1.2 \mathrm{~mm}$, 512 whole brain volumes, FOV $=192 \times 192 \mathrm{~mm}^{2}$, slice acceleration factor: 2 , slice thickness $=1 \mathrm{~mm}, 102$ slices, GRAPPA factor 2, partial Fourier $6 / 8, \mathrm{TR}=1130 \mathrm{~ms}$, $\mathrm{TE}=22 \mathrm{~ms}$, flip angle $=40^{\circ}$, bandwidth $=1562 \mathrm{~Hz} /$ Px] were acquired under an eyes open condition with a fixation cross for $10 \mathrm{~min}$ and took place before the task. Additionally, MP2RAGE images [voxel dimensions $=0.7 \times 0.7 \times 0.7 \mathrm{~mm}, \mathrm{FOV}=224 \times 224 \times 240 \mathrm{~mm}^{3}$, $\mathrm{TR}=5000 \mathrm{~ms}, \mathrm{TE}=2.45 \mathrm{~ms}$, flip angle $1=5^{\circ}$, flip angle $2=3^{\circ}$, bandwidth $=250 \mathrm{~Hz} / \mathrm{Px}$ ] and a fieldmap [voxel dimensions $=2 \times 2 \times 2 \mathrm{~mm}, \mathrm{FOV}=256 \times 256 \mathrm{~mm}^{2}$, slice thickness $=2 \mathrm{~mm}, 80$ slices, $\mathrm{TR}=18 \mathrm{~ms}, \mathrm{TE} 1=4.08 \mathrm{~ms}$, TE2 $=9.18 \mathrm{~ms}$, flip angle $=10^{\circ}$, bandwidth $\left.=300 \mathrm{~Hz} / \mathrm{Px}\right]$ were also collected.

\section{Image processing}

A custom preprocessing pipeline was implemented using Nipype (Gorgolewski et al. 2016) and Nilearn (v0.2.3, Abraham et al. 2014). The first five volumes of the rsfMRI EPI sequences were removed for signal stabilization. Motion correction was performed using the SpaceTimeRealign algorithm (Roche 2011) implemented in Nipy. Given the short TR, no slice timing correction was applied. Magnetic field inhomogeneity and subsequent image distortions are more pronounced at higher field strengths (Cusack et al. 2003), therefore EPI data were undistorted using the fieldmaps and FSL FUGUE (Jenkinson 2004). Outliers in composite motion and average brain signal were detected using Nipype's ArtifactDetect function for removal in a subsequent step. Nilearn's high_variance_confounds function was used to extract time courses representative of physiological noise from the white matter and cerebrospinal fluid, following the aCompCor approach (Behzadi et al. 2007). Nuisance regression was performed using Nilearn's NiftiMasker and included the previously calculated 12 motion regressors ( 3 translations and 3 rotations plus their first derivatives), outlier regressors and physiological regressors. Timeseries were detrended, variance-normalized and bandpass filtered between 0.01 and $0.1 \mathrm{~Hz}$. A $2.4 \mathrm{~mm}$ FWHM Gaussian spatial smoothing filter was applied to the preprocessed data (Poldrack et al. 2011). Eigenvector centrality (EC) maps were calculated with the fast Eigenvector centrality mapping (fastECM) algorithm by Wink et al. (2012) in native space to protect data properties. Finally, a linear (12 degree of freedom (dof)) and nonlinear (Syn) registration to MNI space were performed with ANTS (Avants et al. 2009) as implemented in the CBS Tools (Bazin et al. 2014). Further preprocessing steps such as generating transformation maps between native, group and MNI spaces, as well as tissue segmentation of the anatomical MP2RAGE images were performed using the CBS Tools. All scripts are openly available at https://github.com/AthSchmidt/MMPI/tree/master/preprocessing.

\section{Network analysis}

In recent years, graph-based analyses have become increasingly used for studying functional connectivity (Sami and Miall 2013; Wang et al. 2010 review, Zuo et al. 2012). In such analyses, brain regions are treated as nodes-and several approaches have been used to describe the dynamics of connections between such nodes (Bullmore and Bassett 2011). Graph based analyses also offer the possibility to undertake data-driven investigations of brain dynamics globally and quantifiably on a voxel level without prior assumptions. EC mapping is a method to analyze network structures which identifies nodes that are of central importance to the 
entire network (Lohmann et al. 2010). The importance of nodes is determined by the connectedness to other nodes, increasing as the connection to nodes with many important connections also increases. In other words, nodes become more important to the network when they are connected to other important nodes, and are assigned higher EC values. The weighting of the nodes is achieved by calculating their eigenvector from a similarity matrix. (Lohmann et al. 2010). The dominant eigenvector is the one with the largest eigenvalue of this matrix. The coefficient in this dominant eigenvector is computed iteratively as the weighted sum of centralities of the neighbors of a given node. A detailed description of the calculation can be found in the original article by Wink et al. (2012). Applying fastECM, we analysed node centrality on the whole brain level by treating each voxel as a node. Major advantages of EC compared to other rsfMRI analysis methods are its faster computation times, and the fact that it is data driven and does not require additional assumptions (Wink et al. 2012).

Applying the fastECM Algorithm by Wink et al. (2012), we analysed EC on the whole brain level by treating each voxel as a node. For the analyses, voxel-wise EC maps at each time point were compared between groups to infer connectivity changes as a result of sequence-specific motor learning. For example, the changes between $\mathrm{d} 1$ and $\mathrm{d} 2$ of the LRN group were compared to the changes between $\mathrm{d} 1$ and $\mathrm{d} 2$ of the SMP group in order to identify potential interaction effects. The comparisons between time points were interpreted as being reflective of the different learning stages. Based on the motor learning literature, we defined fast learning as changes between the first and second days of training (d1/d2), slow learning as improvements between the second and fifth days (d2/d5), overall learning from no knowledge of the task up until plateau (d1/d5) and retention as skill recall after the passage of time without training (d5/ d17) (Dayan and Cohen 2011).

\section{Behavioural analysis}

We analysed the behavioural data to characterize performance and contextualize our fMRI results within the different stages of sequence learning introduced by task-based fMRI studies. Performance was assessed by measuring temporal synchronization (SYN) and lag-aligned root mean squared error (RMSE). SYN describes the deviation of the FOR bar from the REF bar, in milliseconds (LRN sequence or SMP sequence). A cross-correlation between REF and FOR patterns was computed for each trial, and the time lag with the maximum correlation value was used to determine the degree of temporal synchronization (SYN), (where a score of 0 denotes perfectly synchronized performance). The lag-aligned values were computed by setting the lag values to 0 . The resulting deviation values were square rooted and averaged. The square root was then used to calculate the lag-aligned RMSE. RMSE represents the spatial deviation between the position of REF and the position of FOR adjusted by the participant. To ensure that they did not differ behaviourally at the beginning of training, we conducted $t$ tests and compared the averages of the first block (three trials) of the SMP sequence in both groups for both SYN and RMSE. Behavioural performance metrics were computed using custom-built MATLAB scripts (available at https:// github.com/neuralabc/SPFT).

\section{Statistical analyses}

In order to understand changes in performance as a result of training, we computed the mean SYN per day for each participant and performed a repeated measures ANOVA with factors day (1-17) and group (LRN vs. SMP). Mauchly's tests were conducted to correct sphericity when necessary (Greenhouse-Geisser if $\varepsilon<0.75$ or Huynh-Feldt if $\varepsilon>0.75$ ). Post hoc Tukey's tests were used to assess timing-specific significant effects between consecutive days. Statistical testing on the behavioural data was performed with the Jamovi software (https://www.jamovi.org; Singmann et al. 2018; Lenth et al. 2018) based on R (https://cran.r-project.org/).

\section{Group X time interaction analyses}

Prior to the interaction analysis and to verify that the groups baseline EC maps did not differ on the days before training, we applied independent $t$ tests in SPM to compare both groups at $\mathrm{d} 0$ as well as $\mathrm{d} 1$.

For the rsFC whole brain interaction analyses we used a flexible factorial design for longitudinal data from the CAT12 Toolbox in SPM with two groups and 5 time points (d0, d1, d2, d5, d17). The flexible factorial design included two factors: group and time point. Within the interaction analyses, changes between time points within-group were compared across groups and learning stages. For example, when investigating the fast learning stage, the interaction analysis consisted of comparing both the $\mathrm{d} 1-\mathrm{d} 2$ contrast (decreases from $\mathrm{d} 1$ to $\mathrm{d} 2$, relative to $\mathrm{LRN}$ ) and the $\mathrm{d} 2-\mathrm{d} 1$ contrast (increases from $\mathrm{d} 1$ to $\mathrm{d} 2$, relative to LRN). Interaction analyses were performed for the following time point contrasts: d1-d2, d2-d1, d2-d5, d5-d2, d1-d5, d5-d1, $\mathrm{d} 5-\mathrm{d} 17$, and $\mathrm{d} 17-\mathrm{d} 5$. Following the identification of interaction effects, we then computed the magnitude of change within the significant ROI in each group to determine which group was driving the effect. Based on our design, the identification of a change in centrality that was greater in the LRN group than the SMP group was considered as a sequence-specific effect. RsfMRI results are reported using cluster inference with the SPM default primary threshold of 
$p<0.001$ and FDR correction at the cluster level at $p<0.05$ (Woo et al. 2014).

\section{Results}

\section{Behavioural results}

Two outliers were excluded from the behavioural data because their values were more than 2 standard deviations away from the mean on 2 days.

There were no differences between the groups on the averages of the first 3 trials of the SMP task in either SYN $(t=-1.84, p=0.07)$ or RMSE $(t=-0.99, p=0.33)$.

For the analysis of SYN, we found a significant main effect of group $\left[\mathrm{F}(2,57)=60.2, p<0.001, \eta^{2}=0.68\right]$ (Fig. 2). Post hoc Tukey $t$ tests revealed significant differences in the
LRN group between $\mathrm{d} 1$ and $\mathrm{d} 2(t=7.89, p<0.001)$ and $\mathrm{d} 2$ and $\mathrm{d} 3(t=4.23, p=0.004)$. There were no significant differences in the LRN group between days 3, 4, 5 and 17 . There were no significant differences between days in the SMP group, supporting the hypothesis that the SMP group was not improving in temporal accuracy. Therefore, we were able to see the progress of learning the LRN sequence over time (Fig. 2) by assessing SYN. For the analysis of RMSE, we found a significant main effect of group $[\mathrm{F}(2,56)=4.90$, $p=0.011, \eta^{2}=0.149$ ] (Fig. 3). Post hoc Tukey $t$ tests revealed significant differences in the LRN group between $\mathrm{d} 1$ and $\mathrm{d} 2$ $(t=4.89, p<0.001)$ and $\mathrm{d} 2$ and $\mathrm{d} 3(t=4.53, p=0.001)$. We also found significant differences in the SMP group between $\mathrm{d} 1$ and $\mathrm{d} 2(t=5.89, p<0.001)$ but no significant differences between $\mathrm{d} 3, \mathrm{~d} 4, \mathrm{~d} 5$ and $\mathrm{d} 17$ providing evidence for performance improvements during the fast learning phase in the SMP group in spatial accuracy.
Fig. 2 Behavioural results. Temporal synchronization (SYN) for for both groups (LRN and SMP) and sequences across all days (d1-d17). Error bars indicate the standard error of the mean. LRN and LRN_SMP values were calculated by averaging $3 \times 3$ trials per block. SMP values were calculated by averaging $6 \times 3$ trials per block

Fig. 3 Behavioural results. Root-mean-squared error (RMSE) for both groups (LRN and SMP) and sequences across all days (d1-d17). Error bars indicate the standard error of the mean. LRN and LRN_SMP values were calculated by averaging $3 \times 3$ trials per block. SMP values were calculated by averaging $6 \times 3$ trials per block
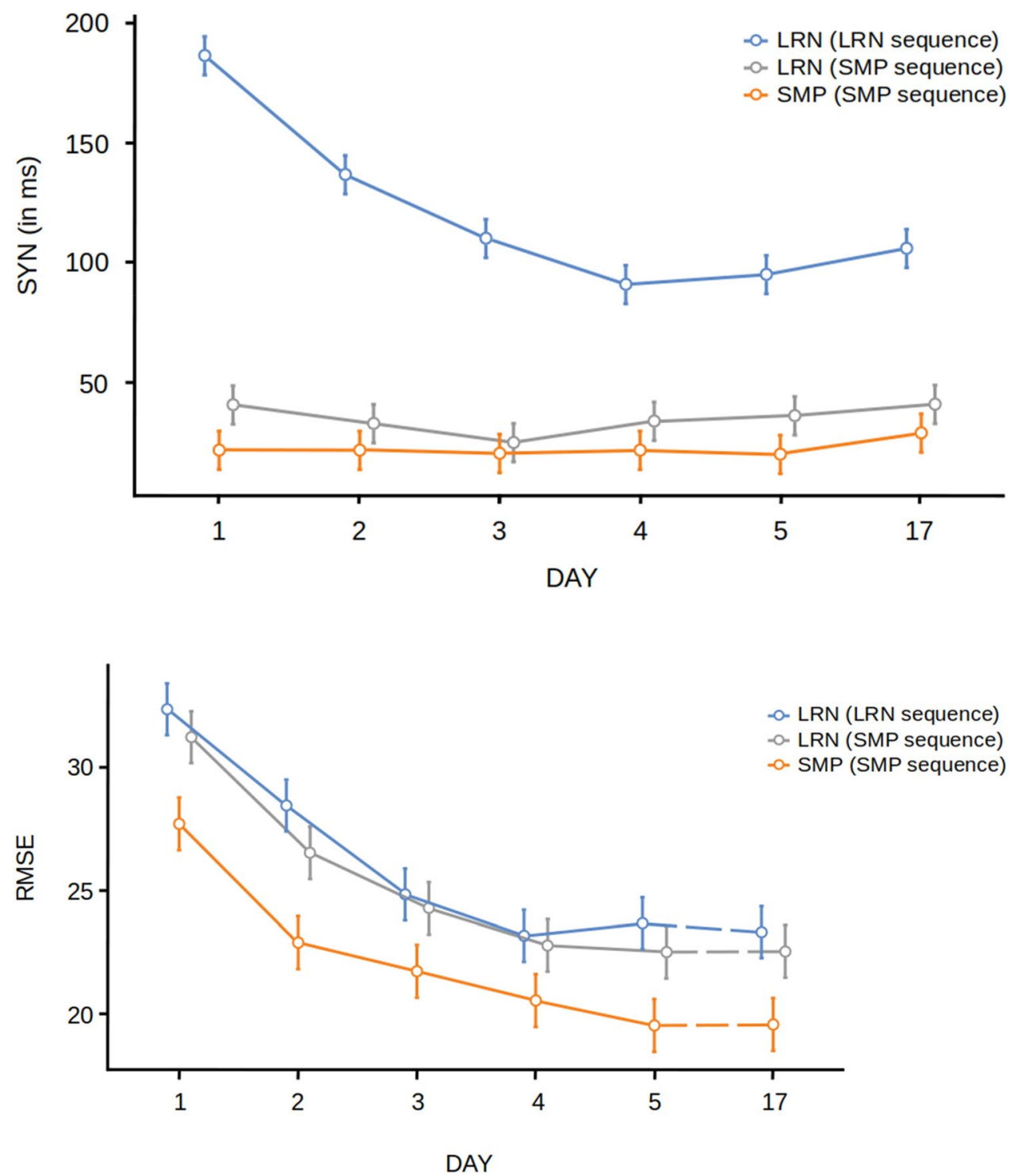
There were no significant differences between the two groups in musical and physical exercise experience (all $p$ values $>0.05$ ). There were no significant differences in the LRN group between days 3, 4, 5 and 17 .

\section{rsFC results}

There were no significant between-group differences in whole brain $\mathrm{EC}$ on either $\mathrm{d} 0$ or $\mathrm{d} 1$.

\section{Interaction effects-fast learning}

When investigating changes in $\mathrm{EC}$ we found significant group by day interaction during fast learning in the right anterior insular cortex (AIC) and the right SPC (Fig. 4). Trajectories of change in EC were plotted over all training days in significant clusters of the interaction analyses to provide additional context about the progression of changes within those regions across the time points (Fig. 4).

\section{Interaction effects-slow learning}

Interaction effects between $\mathrm{d} 2$ and $\mathrm{d} 5$ were found in $\mathrm{EC}$ between groups in the right AIC (Fig. 5). However, this cluster was not sequence specific, as the SMP group was driving the changes.

\section{Interaction effects-overall learning}

We found interaction effects between groups in EC during overall learning (d1 vs d5) in the right supplementary motor area (SMA) and right parietal operculum (PO) (secondary somatosensory cortex) (Fig. 6A) and bilateral SPC (Fig. 6B). Interaction effects in the right SMA were driven by the LRN group (see Table 1) and, therefore, identified
Fig. 4 Group interaction: fast learning. a Glass brain EC. Interaction effect in the right AIC, decreases in LRN (d1-d2), b glass brain EC. Interaction effect in the right SPC, increases in LRN (d2-d1). c The EC change value trajectory over the entire training period for the significant clusters including R AIC and R SPC displayed for LRN and SMP. The period which had the significant interaction $(\mathrm{d} 1 / \mathrm{d} 2)$ is highlighted in red $\mathbf{a}$

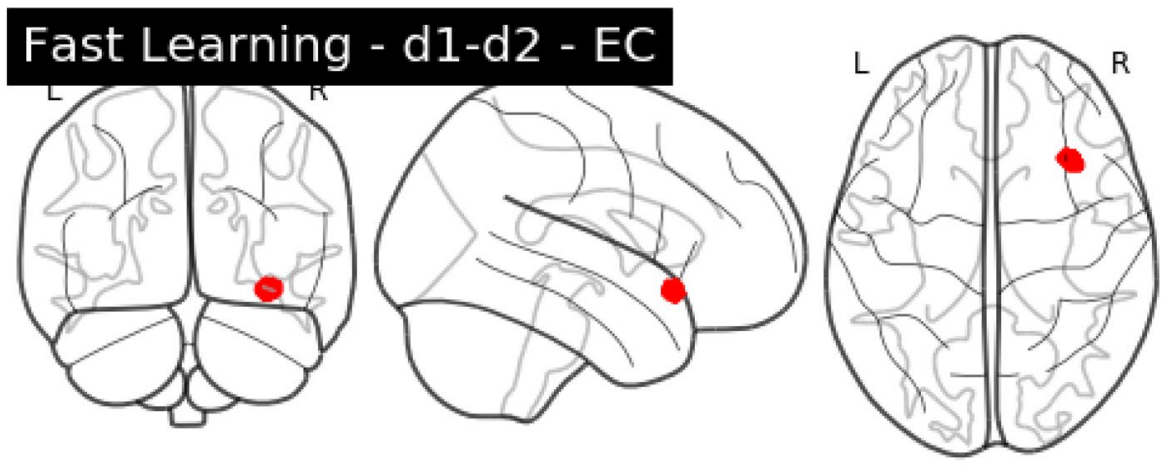

b
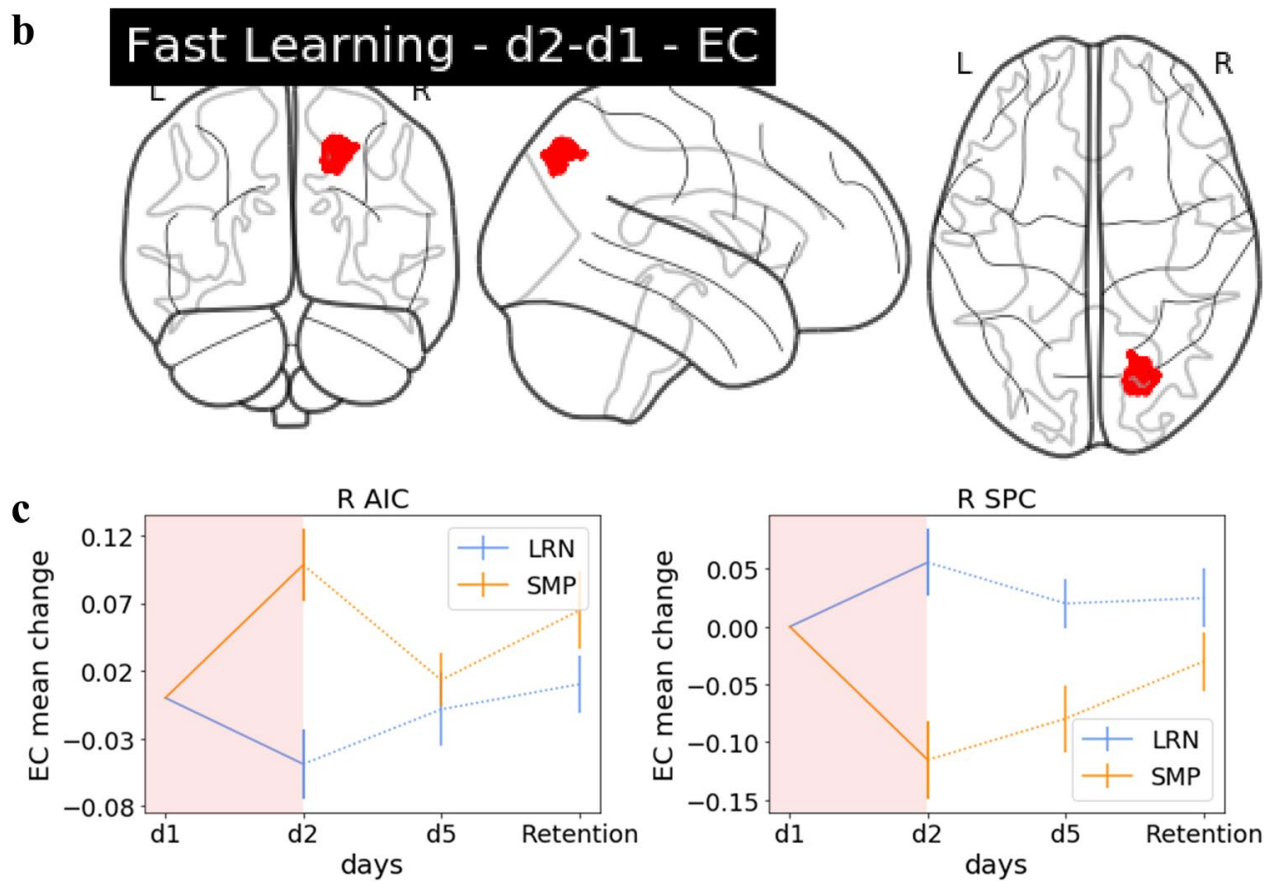
Fig. 5 Group interaction: slow learning. a Glass brain EC. Interaction effects in the R AIC, increases in LRN (d5-d2). b The EC change value trajectory over the entire training period for the significant cluster in $\mathrm{R}$ AIC displayed for LRN and SMP with the period which had the significant interaction (d2/ d5) highlighted in purple a
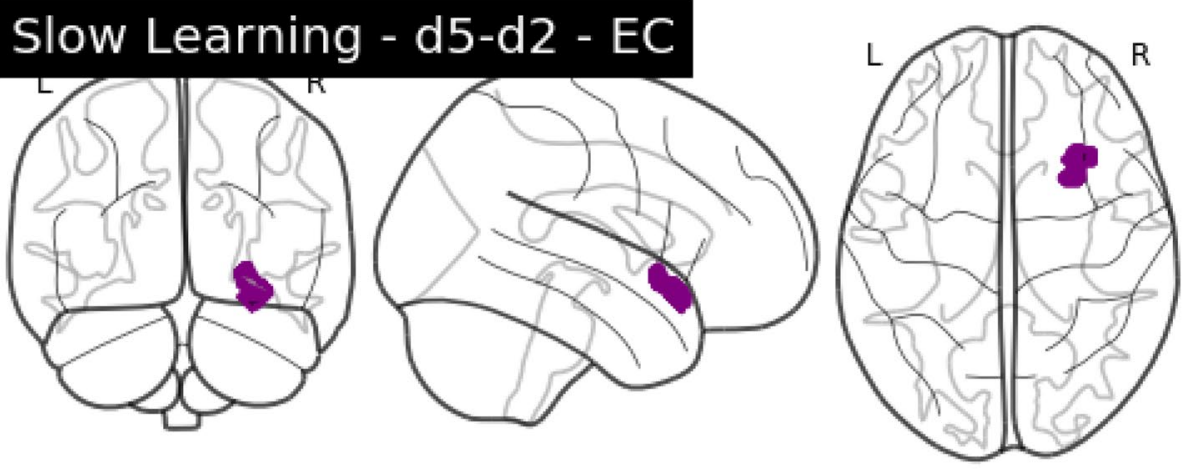

b

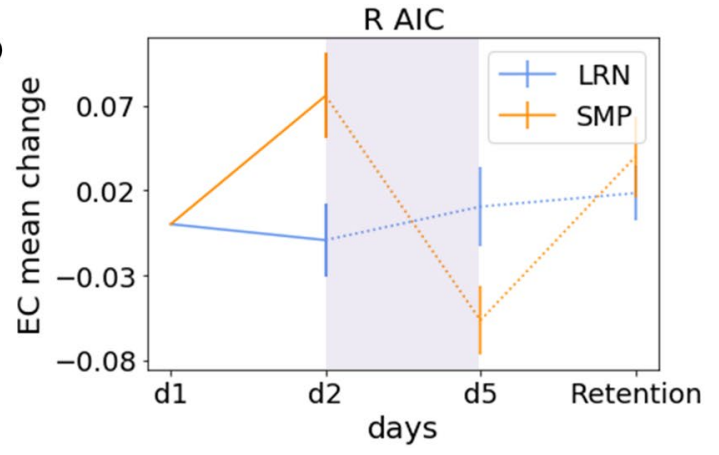

as sequence-specific. A depiction of the precise anatomical location of this cluster in the right SMA can be found in Fig. 7.

\section{Interaction effects-retention}

To identify potential EC changes between the last time point of training and the retention probe, we compared $\mathrm{d} 5$ and $\mathrm{d} 17$ between the two groups. We identified a significant interaction effect in the right Putamen (Fig. 8). This effect was not predominantly driven by LRN.

Precise locations as well as MNI coordinates of peak voxels and significance thresholds of the significant clusters are listed in Table 2.

\section{Discussion}

We investigated between-group interaction effects in functional connectivity changes over the course of one week of training on a continuous motor sequence task with rsfMRI. Our study encompasses fast learning, slow learning, overall learning, and retention. Crucially, we compared a group that learned a complex sequence with a control group that performed a matched motor task to distinguish changes due to sequence-specific learning and provide additional context for changes in resting state functional connectivity after MSL throughout the learning stages.
Interaction analyses revealed a set of regions mostly within the motor network that exhibited differential change between the two groups, providing evidence that these regions play a role in functional plasticity after MSL. Our criterion for assigning sequence-specific function to a region was dependent on that region exhibiting greater centrality change in the LRN group. As a result, we found sequencespecific functional changes in one brain region at one time point: the SMA during overall learning. Most of the other regions identified in the interactional analyses, including well-known motor learning related areas such as the SPC also showed opposing centrality changes in both groups but were not being driven by LRN, suggesting differential involvement of this region when learning a complex motor sequence versus a simpler motor execution.

\section{Sequence specificity}

Behaviourally, we were able to show sequence-specific increases in performance over the course of training. Both behavioural measures showed differential improvements in performance over the course of training, and the experimental and control groups both improved in spatial accuracy (RMSE). Furthermore, both sequences showed similar patterns of improvement in the LRN group, while the control group's performance was consistently better (lower RMSE on the SMP sequence than in the LRN group). This result is likely because we controlled for the amount of motor execution (i.e., the control group performed the same 
Fig. 6 Group interaction: overall learning. a Glass brain EC. Interaction effects in the R PO and R SMA (seq-spec.), decreases in LRN (d1-d5). b Glass brain EC. Interaction effects in bilateral SPC, increases in LRN (d5-d1). c The EC change value trajectory over the entire training period for the significant clusters in R PO, SMA, SPC and L SPC displayed for LRN and SMP with the period which had the significant interaction (d2/d5) highlighted in green $\mathbf{a}$

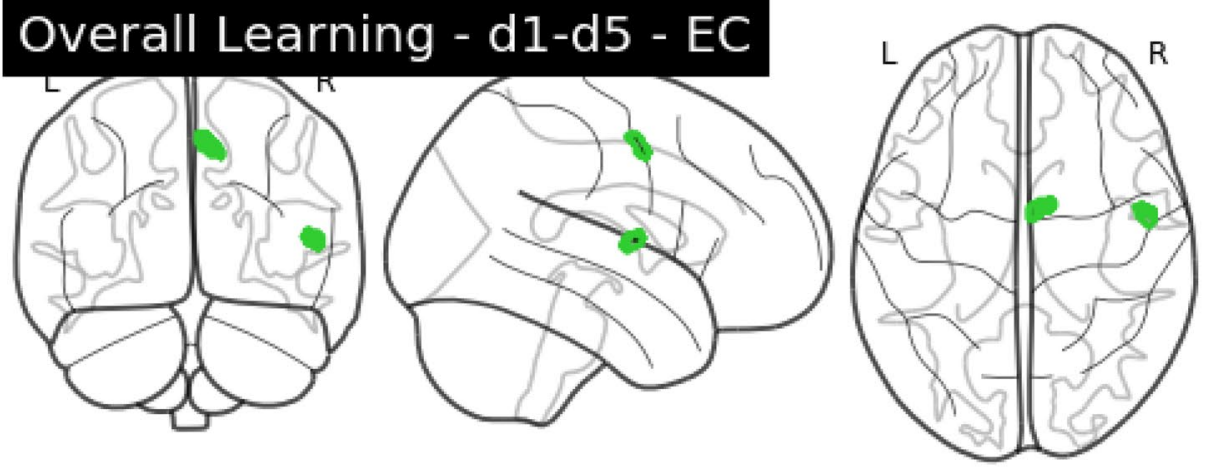

b

Overall Learning - d5-d1 - EC
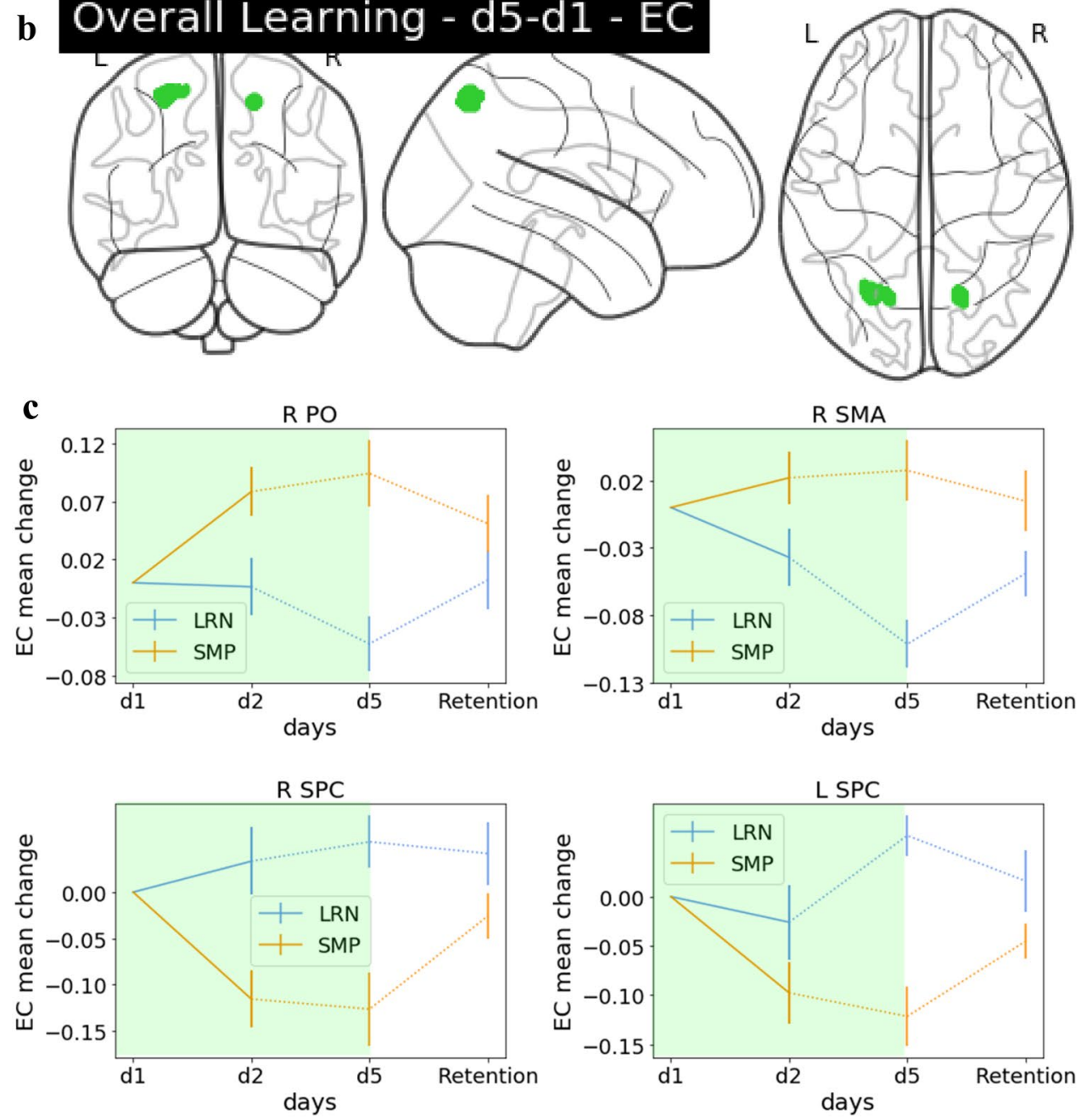

number of sequences as the LRN group, but performed only the SMP sequence. This means that the control group performed twice the number of SMP sequences and was not alternating between sequences as in the LRN group. This design resulted in an overall improvement in spatial accuracy in the LRN group that was less than that of the control group. Regardless, it is apparent that both groups exhibited performance improvements in spatial accuracy that followed very similar trajectories-indicating that the improvement in spatial accuracy is relevant to both groups. However, when measuring temporal accuracy (SYN), we found that behavioural improvements were exclusively evident for the complex LRN sequence. Therefore, our findings indicate that SYN exhibits sequence-specificity and reflects complex 
Table 1 Summary statistics for regions exhibiting significant time-by-group interactions

\begin{tabular}{|c|c|c|c|c|c|c|c|}
\hline \multirow[t]{2}{*}{$\mathrm{EC}$} & & \multicolumn{3}{|l|}{ LRN } & \multicolumn{3}{|l|}{ SMP } \\
\hline & & Mean & SEM & $95 \% \mathrm{CI}$ & Mean & SEM & $95 \% \mathrm{CI}$ \\
\hline \multirow{2}{*}{$\begin{array}{l}\text { Fast learning } \\
(\mathrm{d} 1 \text { vs } \mathrm{d} 2)\end{array}$} & R AIC & -0.05 & 0.03 & {$[-0.10,0.01]$} & 0.10 & 0.03 & {$[0.04,0.16]$} \\
\hline & R SPC & 0.06 & 0.03 & {$[-0.01,0.12]$} & -0.12 & 0.03 & {$[-0.19,-0.04]$} \\
\hline $\begin{array}{l}\text { Slow learning } \\
\text { (d2 vs d5) }\end{array}$ & R AIC & 0.02 & 0.03 & {$[-0.04,0.08]$} & -0.13 & 0.02 & {$[-0.17,-0.10]$} \\
\hline $\begin{array}{l}\text { Retention } \\
\text { (d5 vs d17) }\end{array}$ & $\begin{array}{l}\text { R Puta- } \\
\text { men }\end{array}$ & -0.02 & 0.02 & {$[-0.06,0.03]$} & 0.12 & 0.03 & {$[0.06,0.19]$} \\
\hline \multirow{4}{*}{$\begin{array}{l}\text { Overall learning } \\
\text { (d1 vs d5) }\end{array}$} & R SMA & -0.10 & 0.02 & {$[-0.14,-0.06]$} & 0.03 & 0.02 & {$[-0.02,0.08]$} \\
\hline & $\mathrm{R} P O$ & -0.05 & 0.02 & {$[-0.10,-0.00]$} & 0.09 & 0.03 & {$[0.03,0.16]$} \\
\hline & L SPC & 0.06 & 0.02 & {$[0.02,0.11]$} & -0.12 & 0.03 & {$[-0.19,-0.06]$} \\
\hline & R SPC & 0.05 & 0.03 & {$[-0.01,0.12]$} & -0.13 & 0.04 & {$[-0.21,-0.04]$} \\
\hline
\end{tabular}

Regions where LRN exhibited greater change than SMP are highlighted in grey

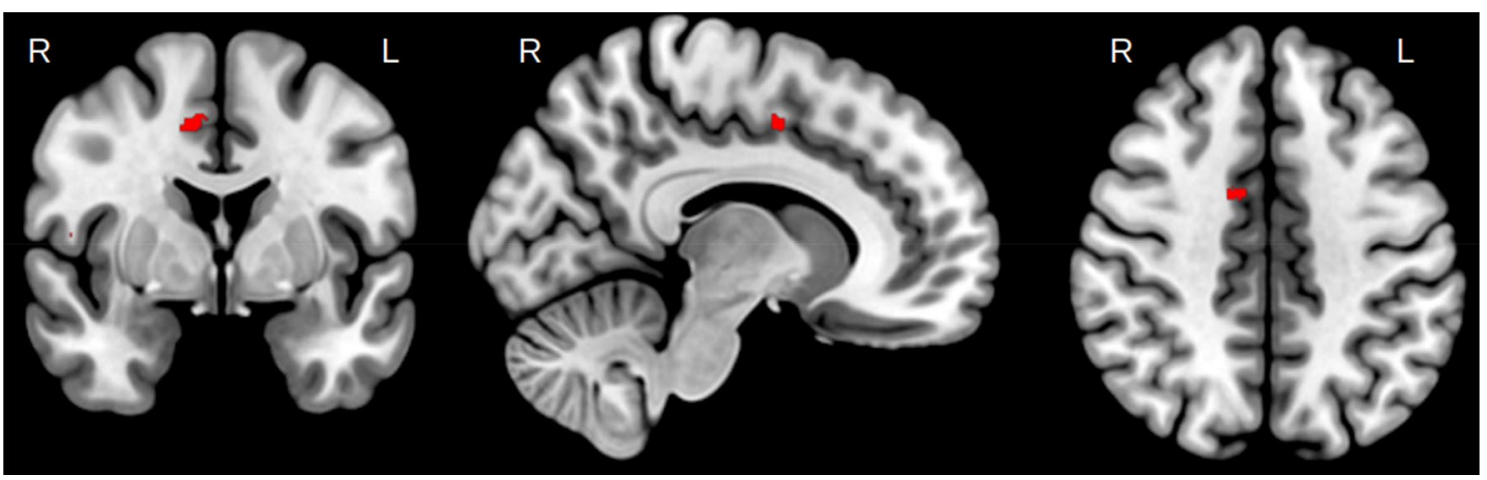

Fig. 7 Anatomical location of the sequence-specific cluster in the R SMA overlaid on an anatomical group template normalized into MNI space

sequence learning. In order to identify which brain regions were relevant for sequence-specific learning and temporal accuracy, we evaluated which of the results from our functional connectivity interaction analyses were reflective of the sequence-specific behavioural results and therefore were 1 . driven by the LRN group and 2. showed little to no change in the SMP group.

Hence, functional connectivity changes reflecting sequence-specific learning effects were identified during the overall learning period in the SMA. We found no evidence for sequence-specific effects during the fast-, slowlearning or retention phases. A predominant role of the SMA in sequence-specificity during offline learning aligns well with the literature on sequence-specificity from task-based online assessments of MSL (Elsinger et al. 2006; Gaymard et al. 1990; Gerloff et al. 1997; Hikosaka et al. 1999; Jenkins et al. 1994; Lee and Quessy 2003; Mushiake et al. 1991; Shibasaki et al. 1993; Shima and Tanji 2000; Tanaka et al. 2010; Vollmann et al. 2013).

Previous research has shown that the SMA is also relevant for sequence-specific MSL during online learning in nonhuman primates where it has been established that SMA is activated during the planning period prior to sequence execution (Tanji and Shima 1994).

While it seems counter-intuitive to see such lateralized results on the right side when training on a motor task performed with the right hand, it is important to consider that the interaction analysis shows results only in regions differing between the experimental and control groups. Considering that both groups are manipulating the device with their right hand, any activity related to simple motor execution will overlap in both groups and, in the case of simple motor movements of the right hand, be present in the left hemisphere. Therefore, sequence-specific differences between groups may be more difficult to detect in these regions as they may end up being masked by connectivity changes attributable to motor execution. Additionally, previous research has shown that the right SMA is bilaterally connected to other regions such as the basal ganglia, insula, thalamus and cerebellum, and thus represents a strong network-level affiliation with the entire motor network (Narayana et al. 2012). The SMA has been found to 
Fig. 8 Group interaction: retention. a Glass brain EC. Interaction effects in the R Putamen, decreases in LRN (d5-d17), b the EC change value trajectory over the entire training period for the significant cluster in $\mathrm{R}$ Putamen displayed for LRN and SMP with the period which had the significant interaction (d5/ d17) highlighted in blue
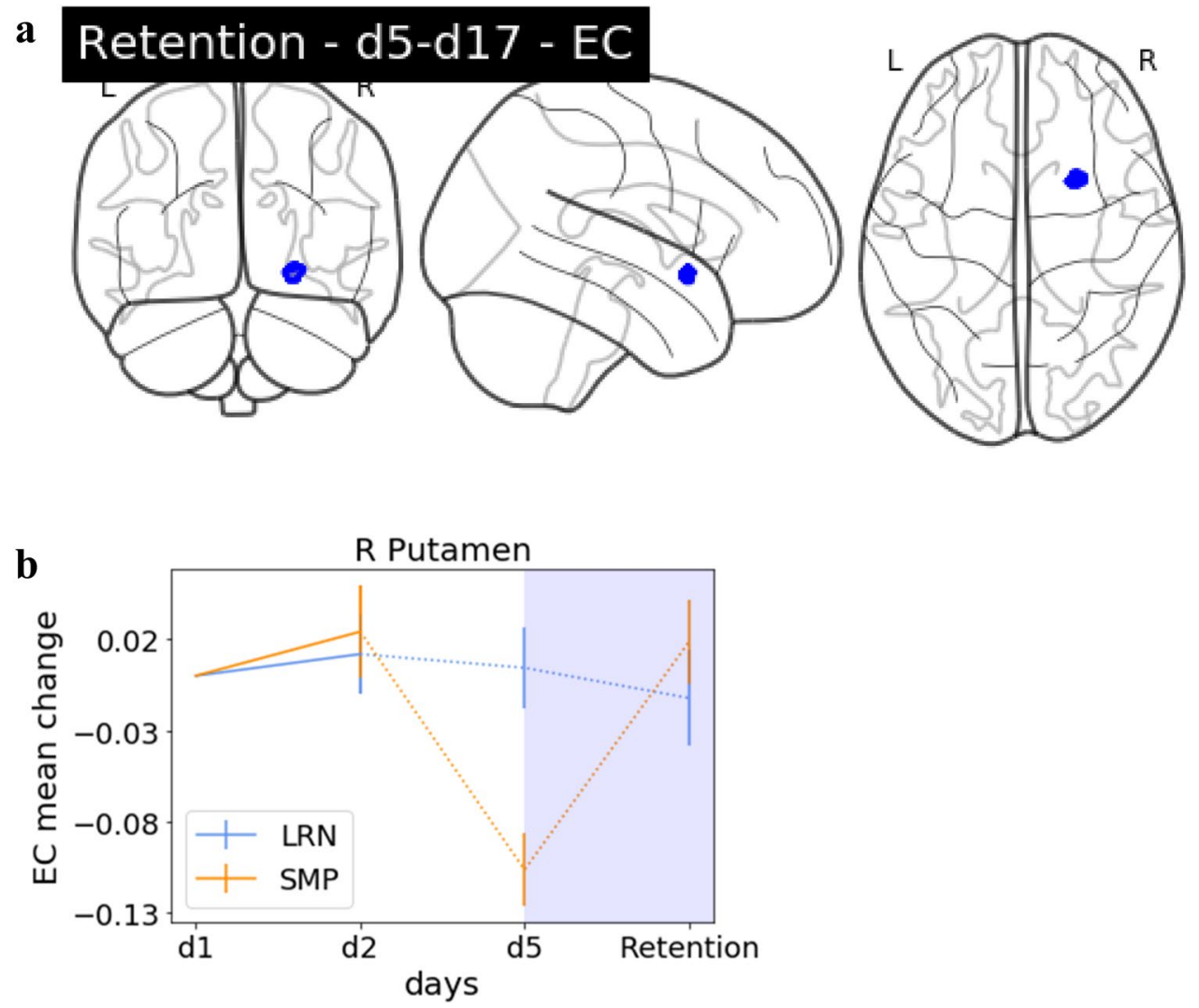

be relevant for sequential learning (Lacourse et al. 2005; Lin et al. 2012; Mallol et al. 2007; Wymbs and Grafton 2015), and specifically to the linking of action phases during sequential movements (Säfström and Domellöf 2018), action execution (Narayana et al. 2012) as well as imagining rhythmic motor execution (Oullier et al. 2005). It is interesting that we identified the right SMA as sequencespecific while previous work has suggested that effectorindependent movement sequences are represented in the left SMA (Grafton et al. 1998, 2002) and Vollmann et al. (2013) showed that tDCS over left SMA promotes learning on the same SPFT that we have used. However, previous

Table 2 Flexible factorial analysis interaction effect results, EC

\begin{tabular}{llllll}
\hline Contrast & pval FDR & K & $\begin{array}{l}\text { Peak } \\
t \text { value }\end{array}$ & $\begin{array}{l}\text { MNI coordi- } \\
\text { nates }\end{array}$ & $\begin{array}{l}\text { Anatomical } \\
\text { location }\end{array}$ \\
\hline $\mathrm{d} 1-\mathrm{d} 2$ & 0.001 & 144 & 4.21 & $35,22,-14$ & R AIC \\
$\mathrm{d} 2-\mathrm{d} 1$ & 0.003 & 431 & 3.88 & $24,-76,47$ & R SPC \\
$\mathrm{d} 5-\mathrm{d} 2$ & 0 & 391 & 4.5 & $30,18,-14$ & R AIC \\
$\mathrm{d} 5-\mathrm{d} 17$ & 0.025 & 27 & 4.25 & $24,10,-10$ & R Putamen \\
$\mathrm{d} 1-\mathrm{d} 5$ & 0 & 131 & 4.03 & $6,-4,52$ & R SMA \\
$\mathrm{d} 1-\mathrm{d} 5$ & 0.002 & 92 & 3.8 & $52,-2,7$ & R PO \\
$\mathrm{d} 5-\mathrm{d} 1$ & 0.008 & 244 & 4.47 & $-26,-66,55$ & L SPC \\
$\mathrm{d} 5-\mathrm{d} 1$ & 0.008 & 88 & 3.81 & $17,-65,52$ & R SPC \\
\hline
\end{tabular}

research has also shown that ipsilateral motor regions play a supportive/cooperative role in learning (Waters et al. 2017), even though their specific involvement in motor plasticity is not fully understood (Hazeltine 2001). One important point made by van Mier et al (1998) was that conclusions on laterality in SMA clusters are often difficult to make with large smoothing kernels (Wymbs and Grafton 2015). Our tightly controlled high-resolution data and small smoothing kernel may provide evidence for a more specific interpretation regarding lateralization. Namely, our results point towards a specific involvement of the right SMA in complex sequence learning a right hand motor task. Whether this involvement is specific to the right hemisphere or rather an ipsilateral effect would need to be investigated in future studies. We found that EC in the SMA gradually decreased over the entire learning period, suggesting an ongoing relevance to the learning process. While it would be intuitive to interpret a decrease in connectivity as indicating decreased involvement of that area, it is important to keep in mind that EC is a network measure that reflects less connectedness to other highly connected nodes, rather than less activation of the region per se. A decrease in this case could also point towards a reduction of neural resources as a result of skill-specific efficiency (Wymbs and Grafton 2015). 


\section{Motor execution}

All clusters identified in the interaction analysis that were not in the right SMA showed differential connectivity changes across both groups. This means that one way or another (increases/decreases) these regions were recruited during training (SMP or LRN). Given that the goal of this study was to identify regions that were exclusively involved in complex sequence learning, we focused on regions that were not involved in simple motor execution and therefore not differentially recruited by the control group. However, it is still the case that other regions that we have identified, though not exhibiting exclusively sequence-specific change, are relevant to both groups and, therefore, likely implicated in motor execution. A comprehensive list of these regions can be found in Table 2 .

Within our interaction analysis, we found significant effects, mostly within the motor network. These effects were driven by the SMP group in bilateral SPC, possibly reflecting the behavioural improvements in spatial accuracy. The SPC, a key node in the motor learning network (Dayan and Cohen 2011; Doyon et al. 2018; Krakauer et al. 2019; Penhune and Steele 2012), showed centrality decreases in SMP. The SPC is involved in learning the association of visual stimuli and motor actions/visuomotor control (Hardwick et al. 2013; Mutha et al. 2011; Müller et al. 2002), and has been found to be relevant for early and later learning stages (Ma et al. 2011). We observed a steady decrease in SMP in functional connectivity in both regions from $\mathrm{d} 1$ to $\mathrm{d} 2$ and $\mathrm{d} 1$ through $\mathrm{d} 5$. Considering that the $\mathrm{d} 1 / \mathrm{d} 2$ comparison reflects fast learning and the $\mathrm{d} 1 / \mathrm{d} 5$ reflects the overall learning process, we propose that the SPC exhibits a slow change in connectivity over the course of the entire learning period resulting in a centrality decrease for SMP. These centrality changes could reflect a continuous learning process that may serve to integrate the presented visual sequence with the required motor response. While it is reasonable to conclude that the SPC is relevant for both LRN and SMP, as the tasks performed by both groups require the coordination of visual and motor information, it is interesting to note that while there are functional changes evident in the LRN group that may be related to the behavioural improvements, they are not as distinct as those in the SMP group.

Interestingly, within our trajectory visualizations, we found that the changes in centrality in most regions showed opposite directionalities. It is possible that these different directionalities of change as well as the differences in magnitude of change could be due to task complexity (Carey et al. 2005; Witt et al. 2008). We speculate that SMP, being a rather simple task, would cause centrality changes to occur faster, such that we are presented with change reflecting a well-learned or overlearned task (motor execution). In contrast, centrality changes in LRN would rather reflect changes during the actual learning process of the complex sequence within our experimental group.

\section{Conclusion}

The present experiment employed a mixed longitudinal training design with two groups (learning and control) to investigate learning on a continuous motor sequence task. We provide evidence for connectivity changes in the right SMA being specific to the learned sequence rather than motor execution. We also argue that changes in resting state centrality in other regions of the motor network, including the SPC, could be due to motor execution processes common to both tasks.

Funding Open Access funding enabled and organized by Projekt DEAL. This work was supported by the Max Planck Society, the MaxNetAging Research School (Max Planck Institute for Demographic Research, ATJ), the NWO Vici grant (PI: Birte Forstmann) (PLB), the Heart and Stroke Foundation of Canada (CJS, CJG), the Michal and Renata Hornstein Chair in Cardiovascular Imaging (CJG), the Natural Science and Engineering Research Council (CJS: RGPIN-2020-06812; CJG: RGPIN 2015-04665) and the Fonds Recherche Québécois Nature et Technologies (CJS).

Data availability Statistical maps will be made available upon reasonable request and released on Neurovault following publication. Due to the ethical standards of the Ethics Committee of the University of Leipzig, raw data cannot be made available.

Code availability The preprocessing pipeline is available at: https:// github.com/AthSchmidt/MMPI/tree/master/preprocessing. Behavioural performance metric computation scripts are available at https://github. com/neuralabc/SPFT.

\section{Declarations}

Ethical approval All study procedures were in accordance with the general ethical standards of the Ethics Committee of the University of Leipzig and in line with the 1964 Helsinki declaration. The study was further approved by the Ethics Committee of the University of Leipzig.

Open Access This article is licensed under a Creative Commons Attribution 4.0 International License, which permits use, sharing, adaptation, distribution and reproduction in any medium or format, as long as you give appropriate credit to the original author(s) and the source, provide a link to the Creative Commons licence, and indicate if changes were made. The images or other third party material in this article are included in the article's Creative Commons licence, unless indicated otherwise in a credit line to the material. If material is not included in the article's Creative Commons licence and your intended use is not permitted by statutory regulation or exceeds the permitted use, you will need to obtain permission directly from the copyright holder. To view a copy of this licence, visit http://creativecommons.org/licenses/by/4.0/. 


\section{References}

Abraham A, Pedregosa F, Eickenberg M, Gervais P, Mueller A, Kossaifi J, Gramfort A, Thirion B, Varoquaux G (2014) Machine learning for neuroimaging with scikit-learn. Front Neuroinform. https://doi.org/10.3389/fninf.2014.00014

Albert NB, Robertson EM, Miall RC (2009) The resting human brain and motor learning. Curr Biol 19(12):1023-1027. https://doi.org/ 10.1016/j.cub.2009.04.028

Avants BB, Tustison N, Song G (2009) Advanced normalization tools (ANTS). Insight J 2:1-35

Bazin P-L, Weiss M, Dinse J, Schäfer A, Trampel R, Turner R (2014) A computational framework for ultra-high resolution cortical segmentation at 7Tesla. Neuroimage 93:201-209. https://doi.org/10. 1016/j.neuroimage.2013.03.077

Behzadi Y, Restom K, Liau J, Liu TT (2007) A component based noise correction method (CompCor) for bold and perfusion based fMRI. Neuroimage 37(1):90-101. https://doi.org/10.1016/j.neuroimage. 2007.04.042

Bengtsson SL, Nagy Z, Skare S, Forsman L, Forssberg H, Ullén F (2005) Extensive piano practicing has regionally specific effects on white matter development. Nat Neurosci 8(9):1148-1150. https://doi.org/10.1038/nn1516

Biswal B, Zerrin Yetkin F, Haughton VM, Hyde JS (1995) Functional connectivity in the motor cortex of resting human brain using echo-planar mri. Magn Reson Med 34(4):537-541. https://doi. org/10.1002/mrm.1910340409

Bullmore ET, Bassett DS (2011) Brain graphs: graphical models of the human brain connectome. Annu Rev Clin Psychol 7(1):113-140. https://doi.org/10.1146/annurev-clinpsy-040510-143934

Carey JR, Bhatt E, Nagpal A (2005) Neuroplasticity promoted by task complexity. Exerc Sport Sci Rev 33(1):24-31

Coynel D, Marrelec G, Perlbarg V, Pélégrini-Issac M, Van de Moortele P-F, Ugurbil K, Doyon J, Benali H, Lehéricy S (2010) Dynamics of motor-related functional integration during motor sequence learning. Neuroimage 49(1):759-766. https://doi.org/10.1016/j. neuroimage.2009.08.048

Cusack R, Brett M, Osswald K (2003) An evaluation of the use of magnetic field maps to undistort echo-planar images. Neuroimage 18(1):127-142. https://doi.org/10.1006/nimg.2002.1281

Dayan E, Cohen LG (2011) Neuroplasticity subserving motor skill learning. Neuron 72(3):443-454. https://doi.org/10.1016/j.neuron. 2011.10.008

Doyon J, Benali H (2005) Reorganization and plasticity in the adult brain during learning of motor skills. Curr Opin Neurobiol 15(2):161-167. https://doi.org/10.1016/j.conb.2005.03.004

Doyon J, Gabitov E, Vahdat S, Lungu O, Boutin A (2018) Current issues related to motor sequence learning in humans. Curr Opin Behav Sci 20:89-97. https://doi.org/10.1016/j.cobeha.2017.11. 012

Elsinger CL, Harrington DL, Rao SM (2006) From preparation to online control: reappraisal of neural circuitry mediating internally generated and externally guided actions. Neuroimage 31(3):11771187. https://doi.org/10.1016/j.neuroimage.2006.01.041

Gaymard B, Pierrot-Deseilligny C, Rivaud S (1990) Impairment of sequences of memory-guided saccades after supplementary motor area lesions. Ann Neurol 28(5):622-626. https://doi.org/10.1002/ ana.410280504

Gerloff C, Corwell B, Chen R, Hallett M, Cohen LG (1997) Stimulation over the human supplementary motor area interferes with the organization of future elements in complex motor sequences. Brain 120:1587-1602. https://doi.org/10.1093/brain/120.9.1587 (Pt 9)

Gorgolewski KJ, Esteban O, Burns C, Ziegler E, Pinsard B, Madison C, Waskom M, Ellis DG, Clark D, Dayan M, Manhães-Savio A,
Notter MP, Johnson H, Dewey BE, Halchenko YO, Hamalainen C, Keshavan A, Clark D, Huntenburg JM, Ghosh S (2016) Nipype: a flexible, lightweight and extensible neuroimaging data processing framework in Python 0.12.0-rc1. Zenodo. https://doi.org/10.5281/zenodo.50186

Grafton ST, Hazeltine E, Ivry RB (1998) Abstract and effector-specific representations of motor sequences identified with PET. J Neurosci 18(22):9420-9428. https://doi.org/10.1523/JNEUR OSCI.18-22-09420.1998

Grafton ST, Hazeltine E, Ivry RB (2002) Motor sequence learning with the nondominant left hand. Exp Brain Res 146(3):369-378. https://doi.org/10.1007/s00221-002-1181-y

Gregory MD, Agam Y, Selvadurai C, Nagy A, Vangel M, Tucker M, Robertson EM, Stickgold R, Manoach DS (2014) Resting state connectivity immediately following learning correlates with subsequent sleep-dependent enhancement of motor task performance. Neuroimage 102(02):666-673. https://doi.org/10. 1016/j.neuroimage.2014.08.044

Gryga M, Taubert M, Dukart J, Vollmann H, Conde V, Sehm B, Villringer A, Ragert P (2012) Bidirectional gray matter changes after complex motor skill learning. Front Syst Neurosci. https:// doi.org/10.3389/fnsys.2012.00037

Guerra-Carrillo B, Mackey AP, Bunge SA (2014) Resting-state fMRI: a window into human brain plasticity. Neuroscientist 20(5):522533. https://doi.org/10.1177/1073858414524442

Hardwick RM, Rottschy C, Miall RC, Eickhoff SB (2013) A quantitative meta-analysis and review of motor learning in the human brain. Neuroimage 67:283-297. https://doi.org/10.1016/j.neuro image.2012.11.020

Hazeltine E (2001) Ipsilateral sensorimotor regions and motor sequence learning. Trends Cogn Sci 5(7):281-282. https://doi. org/10.1016/S1364-6613(00)01702-2

Hikosaka O, Nakahara H, Rand MK, Sakai K, Lu X, Nakamura K, Miyachi S, Doya K (1999) Parallel neural networks for learning sequential procedures. Trends Neurosci 22(10):464-471

Jenkins I, Brooks D, Nixon P, Frackowiak R, Passingham R (1994) Motor sequence learning: a study with positron emission tomography. J Neurosci 14(6):3775-3790. https://doi.org/10.1523/ JNEUROSCI.14-06-03775.1994

Jenkinson M (2004) Improving the registration of B0- distorted EPI images using calculated cost function weights. 10th International Conference on Functional Mapping of the Human Brain

Karim HT, Huppert TJ, Erickson KI, Wollam ME, Sparto PJ, Sejdić E, VanSwearingen JM (2017) Motor sequence learning-induced neural efficiency in functional brain connectivity. Behav Brain Res 319:87-95. https://doi.org/10.1016/j.bbr.2016.11.021

Krakauer JW, Hadjiosif AM, Xu J, Wong AL, Haith AM (2019) Motor learning. Compr Physiol 9(2):613-663. https://doi.org/ 10.1002/cphy.c170043

Lacourse MG, Orr ELR, Cramer SC, Cohen MJ (2005) Brain activation during execution and motor imagery of novel and skilled sequential hand movements. Neuroimage 27(3):505-519. https://doi.org/10.1016/j.neuroimage.2005.04.025

Lee D, Quessy S (2003) Activity in the supplementary motor area related to learning and performance during a sequential visuomotor task. J Neurophysiol 89(2):1039-1056. https://doi.org/ 10.1152/jn.00638.2002

Lenth R, Singmann H, Love J, Buerkner P, Herve M (2018) Emmeans: estimated marginal means, aka least-squares means. R Package Version 1(1):3

Lewis CM, Baldassarre A, Committeri G, Romani GL, Corbetta M (2009) Learning sculpts the spontaneous activity of the resting human brain. Proc Natl Acad Sci 106(41):17558-17563. https:// doi.org/10.1073/pnas.0902455106

Lin (Janice) C-H, Chiang M-C, Wu AD, Iacoboni M, Udompholkul P, Yazdanshenas O, Knowlton BJ (2012) Age related differences 
in the neural substrates of motor sequence learning after interleaved and repetitive practice. Neuroimage 62(3):2007-2020. https://doi.org/10.1016/j.neuroimage.2012.05.015

Lohmann G, Margulies DS, Horstmann A, Pleger B, Lepsien J, Goldhahn D, Schloegl H, Stumvoll M, Villringer A, Turner R (2010) Eigenvector centrality mapping for analyzing connectivity patterns in fMRI data of the human brain. PLoS ONE 5(4):e10232. https://doi.org/10.1371/journal.pone.0010232

Lohse KR, Wadden K, Boyd LA, Hodges NJ (2014) Motor skill acquisition across short and long time scales: a meta-analysis of neuroimaging data. Neuropsychologia 59:130-141. https:// doi.org/10.1016/j.neuropsychologia.2014.05.001

Ma L, Narayana S, Robin DA, Fox PT, Xiong J (2011) Changes occur in resting state network of motor system during 4 weeks of motor skill learning. Neuroimage 58(1):226-233. https://doi. org/10.1016/j.neuroimage.2011.06.014

Mallol R, Barrós-Loscertales A, López M, Belloch V, Parcet MA, Ávila C (2007) Compensatory cortical mechanisms in Parkinson's disease evidenced with fMRI during the performance of pre-learned sequential movements. Brain Res 1147:265-271. https://doi.org/10.1016/j.brainres.2007.02.046

Marques JP, Kober T, Krueger G, van der Zwaag W, Van de Moortele P-F, Gruetter R (2010) MP2RAGE, a self bias-field corrected sequence for improved segmentation and T1-mapping at high field. NeuroImage 49(2):1271-1281. https://doi.org/10.1016/j. neuroimage.2009.10.002

Mary A, Wens V, Op de Beeck M, Leproult R, De Tiège X, Peigneux $P$ (2017) Resting-state functional connectivity is an agedependent predictor of motor learning abilities. Cereb Cortex (new York, NY) 27(10):4923-4932. https://doi.org/10.1093/ cercor/bhw286

Müller R-A, Kleinhans N, Pierce K, Kemmotsu N, Courchesne E (2002) Functional MRI of motor sequence acquisition: effects of learning stage and performance. Cogn Brain Res 14(2):277-293. https://doi.org/10.1016/S0926-6410(02)00131-3

Mushiake H, Inase M, Tanji J (1991) Neuronal activity in the primate premotor, supplementary, and precentral motor cortex during visually guided and internally determined sequential movements. J Neurophysiol 66(3):705-718. https://doi.org/10.1152/jn.1991.66.3.705

Mutha PK, Sainburg RL, Haaland KY (2011) Left parietal regions are critical for adaptive visuomotor control. J Neurosci 31(19):69726981. https://doi.org/10.1523/JNEUROSCI.6432-10.2011

Narayana S, Laird AR, Tandon N, Franklin C, Lancaster JL, Fox PT (2012) Electrophysiological and functional connectivity of the human supplementary motor area. Neuroimage 62(1):250-265. https://doi.org/10.1016/j.neuroimage.2012.04.060

Oullier O, Jantzen KJ, Steinberg FL, Kelso JAS (2005) Neural substrates of real and imagined sensorimotor coordination. Cereb Cortex 15(7):975-985. https://doi.org/10.1093/cercor/bhh198

Penhune VB, Steele CJ (2012) Parallel contributions of cerebellar, striatal and M1 mechanisms to motor sequence learning. Behav Brain Res 226(2):579-591. https://doi.org/10.1016/j.bbr.2011.09.044

Poldrack R, Mumford J, Nichols T (2011) Handbook of functional MRI data analysis. Cambridge University Press, Cambridge

Roche A (2011) A four-dimensional registration algorithm with application to joint correction of motion and slice timing in fMRI. IEEE Trans Med Imaging 30(8):1546-1554. https://doi.org/10. 1109/TMI.2011.2131152

Rosenbaum DA, Kenny SB, Derr MA (1983) Hierarchical control of rapid movement sequences. J Exp Psychol 9(1):86-102

Säfström D, Domellöf E (2018) Brain activations supporting linking of action phases in a sequential manual task. Neuroimage 172:608-619. https://doi.org/10.1016/j.neuroimage.2018.02.014

Sami S, Miall RC (2013) Graph network analysis of immediate motorlearning induced changes in resting state BOLD. Front Hum Neurosci. https://doi.org/10.3389/fnhum.2013.00166
Sami S, Robertson EM, Miall RC (2014) The time course of taskspecific memory consolidation effects in resting state networks. J Neurosci 34(11):3982-3992. https://doi.org/10.1523/JNEUR OSCI.4341-13.2014

Scholz J, Klein MC, Behrens TEJ, Johansen-Berg H (2009) Training induces changes in white-matter architecture. Nat Neurosci 12(11):1370-1371. https://doi.org/10.1038/nn.2412

Seidler RD, Purushotham A, Kim S-G, Uğurbil K, Willingham D, Ashe J (2002) Cerebellum activation associated with performance change but not motor learning. Science 296(5575):2043-2046. https://doi.org/10.1126/science.1068524

Shibasaki H, Sadato N, Lyshkow H, Yonekura Y, Honda M, Nagamine T, Suwazono S, Magata Y, Ikeda A, Miyazaki M, Fukuyama H, Asato R, Konishi J (1993) Both primary motor cortex and supplementary motor area play an important role in complex finger movement. Brain 116(6):1387-1398. https://doi.org/10.1093/ brain/116.6.1387

Shima K, Tanji J (2000) Neuronal activity in the supplementary and presupplementary motor areas for temporal organization of multiple movements. J Neurophysiol 84(4):2148-2160. https://doi.org/ 10.1152/jn.2000.84.4.2148

Singmann H, Bolker B, Westfall J, Aust F (2018) afex: analysis of factorial experiments. $\mathrm{R}$ package version $0.20-2$

Steel A, Thomas C, Trefler A, Chen G, Baker CI (2019) Finding the baby in the bath water-evidence for task-specific changes in resting state functional connectivity evoked by training. Neuroimage 188:524-538. https://doi.org/10.1016/j.neuroimage.2018.12.038

Steele CJ, Penhune VB (2010) Specific increases within global decreases: a functional magnetic resonance imaging investigation of five days of motor sequence learning. J Neurosci 30(24):83328341. https://doi.org/10.1523/JNEUROSCI.5569-09.2010

Tanaka S, Honda M, Hanakawa T, Cohen LG (2010) Differential contribution of the supplementary motor area to stabilization of a procedural motor skill acquired through different practice schedules. Cereb Cortex (new York, NY) 20(9):2114-2121. https://doi.org/ 10.1093/cercor/bhp276

Tanji J, Shima K (1994) Role for supplementary motor area cells in planning several movements ahead. Nature 371(6496):413-416. https://doi.org/10.1038/371413a0

Taubert M, Villringer A, Ragert P (2012) Learning-related gray and white matter changes in humans: an update. Neuroscientist 18(4):320-325. https://doi.org/10.1177/1073858411419048

Thomas C, Baker CI (2013) Teaching an adult brain new tricks: a critical review of evidence for training-dependent structural plasticity in humans. Neuroimage 73:225-236. https://doi.org/10.1016/j. neuroimage.2012.03.069

Vahdat S, Darainy M, Milner TE, Ostry DJ (2011) Functionally specific changes in resting-state sensorimotor networks following motor learning. J Neurosci 31(47):16907-16915. https://doi.org/10. 1523/JNEUROSCI.2737-11.2011

van Mier H, Tempel LW, Perlmutter JS, Raichle ME, Petersen SE (1998) Changes in brain activity during motor learning measured with PET: effects of hand of performance and practice. J Neurophysiol 80(4):2177-2199. https://doi.org/10.1152/jn.1998.80.4.2177

Vollmann H, Conde V, Sewerin S, Taubert M, Sehm B, Witte OW, Villringer A, Ragert P (2013) Anodal transcranial direct current stimulation (tDCS) over supplementary motor area (SMA) but not pre-SMA promotes short-term visuomotor learning. Brain Stimul 6(2):101-107. https://doi.org/10.1016/j.brs.2012.03.018

Wang J, Zuo X, He Y (2010) Graph-based network analysis of resting-state functional MRI. Front Syst Neurosci. https://doi.org/10. 3389/fnsys. 2010.00016

Waters S, Wiestler T, Diedrichsen J (2017) Cooperation not competition: bihemispheric tDCS and fMRI show role for ipsilateral hemisphere in motor learning. J Neurosci 37(31):7500-7512. https:// doi.org/10.1523/JNEUROSCI.3414-16.2017 
Wiestler T, Diedrichsen J (2013) Skill learning strengthens cortical representations of motor sequences. Elife 2:e00801. https://doi. org/10.7554/eLife.00801

Wink AM, de Munck JC, van der Werf YD, van den Heuvel OA, Barkhof F (2012) Fast eigenvector centrality mapping of voxel-wise connectivity in functional magnetic resonance imaging: implementation, validation, and interpretation. Brain Connect 2(5):265274. https://doi.org/10.1089/brain.2012.0087

Witt ST, Laird AR, Meyerand ME (2008) Functional neuroimaging correlates of finger-tapping task variations: an ALE meta-analysis. Neuroimage 42(1):343-356. https://doi.org/10.1016/j.neuroimage.2008.04.025

Woo C-W, Krishnan A, Wager TD (2014) Cluster-extent based thresholding in fMRI analyses: pitfalls and recommendations. Neuroimage 91:412-419. https://doi.org/10.1016/j.neuroimage.2013.12.058

Wymbs NF, Grafton ST (2015) The human motor system supports sequence-specific representations over multiple training-dependent timescales. Cereb Cortex (new York, NY) 25(11):4213-4225. https://doi.org/10.1093/cercor/bhu144
Xiong J, Ma L, Wang B, Narayana S, Duff EP, Egan GF, Fox PT (2009) Long-term motor training induced changes in regional cerebral blood flow in both task and resting states. Neuroimage 45(1):75-82. https://doi.org/10.1016/j.neuroimage.2008. 11.016

Yokoi A, Diedrichsen J (2019) Neural organization of hierarchical motor sequence representations in the human neocortex. Neuron. https://doi.org/10.1016/j.neuron.2019.06.017

Zuo X-N, Ehmke R, Mennes M, Imperati D, Castellanos FX, Sporns O, Milham MP (2012) Network centrality in the human functional connectome. Cereb Cortex (new York, NY) 22(8):1862-1875. https://doi.org/10.1093/cercor/bhr269

Publisher's Note Springer Nature remains neutral with regard to jurisdictional claims in published maps and institutional affiliations. 